\title{
High-Resolution Thermal Imaging Methodology for Non-Destructive Evaluation of Historic Structures
}

\author{
Michael Hess ${ }^{\mathrm{a}}$, David Vanoni ${ }^{\mathrm{b}}$, Vid Petrovic ${ }^{\mathrm{b}}$, and Falko Kuester ${ }^{\mathrm{a}}$ \\ ${ }^{a}$ Department of Structural Engineering \\ University of California, San Diego \\ 9500 Gilman Drive, La Jolla, CA 92093 \\ ${ }^{b}$ Department of Computer Science and Engineering \\ University of California, San Diego \\ 9500 Gilman Drive, La Jolla, CA 92093 \\ email: \{mrhess*,dvanoni,vipetrov, fkuester\}@ucsd.edu \\ * Corresponding Author
}




\section{Abstract}

This paper presents a methodology for automated, portable thermography, for the acquisition of high-resolution thermal image mosaics supporting the non-destructive evaluation of historic structures. The presented approach increases the spatial resolution of thermal surveys to a level of detail needed for building scale analysis. The integration of a robotic camera platform enables automated alignment of multiple images into a high-resolution thermal image mosaic giving a holistic view of the structure while maintaining a level of detail equaling or exceeding that of traditional spot surveys using existing cameras. Providing a digital workflow for automated data and metadata recording increases the consistency and accuracy of surveys regardless of the location or operator. An imaging workflow and instrumentation are shown for a case-study on buildings in Florence, Italy demonstrating the effectiveness of this methodology for structural diagnostics.

\section{Keywords: non-destructive evaluation, thermography, structural evaluation, high resolution imaging}

\section{Research Aims}

Thermography is a widely used technology in many disciplines, but there are still limitations in image resolution when it comes to diagnostic surveys on the scale of buildings. This paper presents a methodology that leverages a low-cost, automated portable thermography (APT) system, designed to increase the spatial resolution of thermal images by performing rapid acquisition of thermal image mosaics. The target application for the APT 
methodology is passive building-scale surveys at cultural heritage sites aiming to assist in monitoring energy losses, mapping areas of moisture, revealing structural features, and detecting damage such as delamination and cracking. Automating the process is a step towards a standardized methodology that provides consistency and accuracy in the acquired data and metadata independent of site or operator. The APT workflow aims to be repeatable, provide the necessary setup to ensure accuracy, as well as save time on acquisition and post-processing. The methodology, composed of the APT instrumentation, its sensors, workflow, and imaging results are presented as part of a case study conducted on buildings in Florence, Italy.

\section{Introduction}

Building-scale measurement and analysis are rapidly growing application domains, driven by the swift decay of our built-infrastructure. To address this growing need, instruments, techniques and methodologies for the surface and volumetric assessment of the geometric properties of a structure in combination with material characterization are necessary to create an accurate baseline for subsequent structural health assessments. Common techniques

include photography and photogrammetry, light detection and ranging (LiDAR) for establishing geometry, sub-surface and volumetric imaging techniques such as thermography, ultrasound, ground-penetrating radar (GPR) and x-ray radiography. Thermography, which captures a combination of surface and subsurface information, can measure spatio-temporal ambient signals, from a distance, accomplishing multiple non-destructive evaluation (NDE) objectives. Common application domains of this multi-purpose diag- 
nostics tool include energy audits $[1,2]$ which establish the thermal envelope of a structure, detection of pests, moisture, and mold [3], structural deficiency and integrity assessment focused on detecting cracks, delamination, as well as material characterization. The ability to characterize materials can aid in creating a baseline as-built record showing construction details and modifications of structures. This can be particularly useful when surveying historic structures because they often lack these construction records.

Building-scale structural health assessment requires accurate and detailed measurements at the macro and micro scale, providing a holistic view of the structure, establishing the overall context, as well as the data needed to study small scale characteristics, deficiencies and damage patterns. Given the necessary resolution, instrumentation, time complexity and cost this is rarely done for thermographic surveys. The most advanced thermal cameras currently only achieve one megapixel resolution [4] and they come with a high price tag. When used in the field at building scale, different lenses may be used, a wide field of view lens providing context, but no detail, and a narrow field of view lens providing detail, but no context. With the available time budgets for site surveys, this often results in just a few spot surveys being performed.

In order to mitigate the issue of limited camera resolution, a methodology and optimized workflow are required to facilitate the rapid acquisition of building-scale thermal images. To fill a need for gigapixel imaging robotic camera platforms have emerged [5] to aid in acquisition and stitching of spatially accurate digital image panoramas. Now with the idea being wellestablished for digital photography, it seems reasonable that this approach 
should also extend to thermography to address its limitations in spatial resolution.

The purpose of this work is to highlight how high-resolution thermal data can be accurately acquired for large surface areas using the proposed methodological approach. The methods for interpretation of the data will not change, but the quality of the data will be much higher allowing for more precise analysis conducted by domain experts. The presented methodology is repeatable regardless of the user, and the workflow ensures accuracy and consistent post-processing of the images using recorded metadata.

\section{Related Work}

Infrared thermography is an imaging technique that measures the radiation emitted by a surface in the long wave infrared range of the electromagnetic spectrum. Infrared waves have longer wavelengths than visible light, ranging from 0.78 to 1000 micrometers [6], but can be captured with infrared cameras and subsequently displayed in the visible spectrum. Infrared cameras that are sensitive to different wavelengths exist and wavelengths ranging from 7 to 14 micrometers are typically captured with thermographic camera sensors. These wavelengths shift with the temperature of the surface where the observed infrared wavelength decreases as temperature increases. When the emissive properties of the surface are known the camera can be calibrated to convert the observed wavelengths to emitted temperature. The set of recorded temperatures is then mapped to a range of colors in the visible spectrum in order to display the results against a scale that correlates each color to a temperature value. 
Thermography is classified into two categories, passive thermography and active thermography. Passive thermography uses the ambient or natural heating and cooling of the specimen to measure differences in heat transfer. On the other hand, active thermography utilizes a controlled external heating or cooling source in order to record the heat flux, or rate of thermal energy transfer, across the target surface [6]. The main difference in the two methods is that passive thermography is a snapshot that gives a qualitative evaluation of the target structure while active thermography is a sequence of images that can also yield quantitative results because the external heat source is defined with a known time and strength [7]. For example, with a controlled heat source, the material and structural characteristics of the specimen can be calculated [8].

\subsection{Thermography for Structural Evaluation}

In structural engineering, passive thermography is the preferred technique to use in-situ because it is not practical to have external sources that apply heat evenly to entire surfaces at building scale. In these areas of application, thermography can be used to monitor energy losses, map areas of moisture, and detect delamination and cracks. These inspection techniques are well documented $[1,3,9,10,11]$ and extremely important, especially as structures age; it is crucial to monitor structures to detect damage and flaws early and take corrective actions before the damage has progressed or significant energy is wasted. Thermography presents a non-destructive approach for detecting warning signs and identifying issues that may not be visible to the human eye [11]. A fast thermal surveying methodology will be vital to efficiently monitor the natural aging and decay of the entire built infrastructure. 
One of the most important applications for thermal imaging in NDE is detecting structural details and damage. Grinzato et al. [3, 10], Hess et al. [12], and Imposa [13] explain how thermal image mosaics can be acquired and used towards determining what types of modifications have occurred over the history of the structure. Grinzato outlines the importance of knowing the history of structural modifications and any anomalies within the structure in general, highlighting that accurate structural evaluation and identification of construction details is vital for a proper conservation plan [3]. Thermal imaging is extremely useful in these situations because it is a non-intrusive technique that reveals information about features that are not visible without opening walls or damaging the existing structure in any way.

Balaras et al. [1] explain how thermography can be used in the context of building diagnostics, specifically looking at building energy audits. This application uses thermography to detect areas where heating or cooling is escaping, insulation is missing, or areas where thermal bridges exist. All of these findings are then reported in order to develop a plan to address the issues that were found. The examples in [1] reveal some detail in the thermograms, but no context for the area. With the presented methodology, the whole context can be displayed while maintaining the same level of detail within the high resolution thermal image. The presented approach also allows for the creation of important baseline records for evaluating a building's thermal efficiency and pinpointing sources of heat flux losses and other undesirable environmental outcomes. 


\section{APT Methodology}

The APT methodology, consisting of the instrument and workflow, seeks to create a fast, high-resolution, low-cost approach that automatically acquires, corrects, and processes geo-located, temporally anchored, buildingscale thermal surveys.

\subsection{Proposed APT Instrumentation Requirements}

The instrument consists of a robotic platform and remote imaging payload modules. The platform controls the imaging payload orientation with the ability to measure data such as position, heading, acceleration and vibration, humidity, and ambient temperature. The platform and the imaging payloads are responsible for the acquisition of the thermal images, while the additional sensor measurements from the platform enhance accurate post-processing, analysis, and metadata creation used to geospatially anchor the survey. For example, a GPS sensor can be utilized to geospatially locate the imaging data and a digital compass sensor used to provide the heading of the surface being imaged. Camera movement and vibrations can also be inferred from accelerometers placed on the platform, which can inform automated stitching of the images in post-processing stages and suggest if images are out of focus when captured. The efficiency of the system depends on the resolution of the imaging device, the presence of a focusing motor and the sensitivity of the thermal imaging sensors and robotic platform motors. Requirements and performance of the imaging system will depend on the application and the practitioner's available technology, but the methodology should remain effective. 

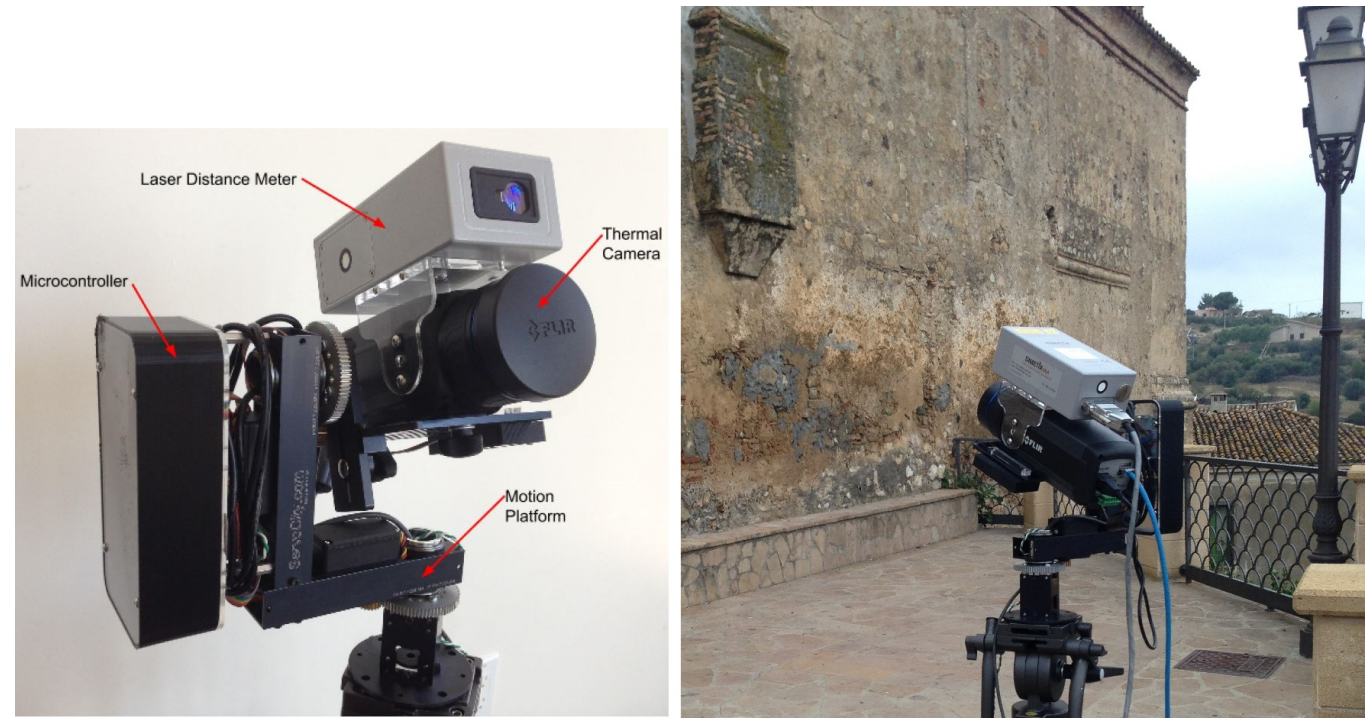

Figure 1: Annotated APT instrument (left) and the APT instrument deployed in the field (right)

With the parameters of the instrumentation well defined, the control system should offer a user interface that aids in the automated image acquisition as well as the storage of metadata relevant to the survey. Metadata can include the name of the project, file names, distance from the structure, time of day the image was taken, air temperature, etc. Automated metadata recording saves the surveyor's time recording data separately and assures a consistent digital workflow. Most importantly, the system must be robust and portable so it can easily be moved and operated on-site.

\subsection{Implemented APT Instrumentation}

The implemented APT imaging payload and robotic platform are shown in Figure 1. The main component of the imaging payload is a GigE Vision compliant FLIR A615 thermal camera [4] and the second component is a 
Table 1: Implemented Instrumentation Specifications

Thermal Camera: FLIR A615 [15]

\begin{tabular}{ll}
\hline IR Resolution & $640 \times 480$ pixels \\
Thermal Sensitivity & $<0.05^{\circ} \mathrm{C} @+30^{\circ} \mathrm{C} / 50 \mathrm{mK}$ \\
Spectral Range & $7.5-14 \mathrm{\mu m}$ \\
Accuracy & $\pm 2^{\circ} \mathrm{C} \mathrm{or} \pm 2 \%$ of reading \\
Field of View & $15^{\circ} \times 11^{\circ}\left(19^{\circ}\right.$ diagonal $)$ \\
Focal Length & $41.3 \mathrm{~mm}$ \\
Acquisition Rate & $50 \mathrm{~Hz}$ \\
Laser Distance Meter: Dimetix AG FLS-C10 $[14]$ \\
Range & $0.05-65 \mathrm{~m}$ \\
Accuracy & $\pm 1 \mathrm{~mm}$ \\
Repeatability & $\pm 0.3 \mathrm{~mm}$ \\
Acquisition Rate & $200 \mathrm{~Hz}$
\end{tabular}

Dimetix AG FLS-C10 [14] laser distance sensor which is mounted above the camera in order to measure accurate distances at every camera position (see Table 1 for specifications). The distance measurements are used to focus the thermal camera properly and provide additional information about the position of the instrumentation setup. The motion platform is a pan and tilt system (PT785-S from ServoCity) driven by two servos and controlled with an Arduino Uno R3 microcontroller board. The implemented robotic platform allows for full $360^{\circ}$ panning and $180^{\circ}$ tilting enabling acquisition of a spherical panorama. The entire APT assembly is then mounted on a tripod for stationary stability and leveling. The presented system uses MATLAB for controlling image acquisition and user input because of its ability to communicate with GigE Vision cameras. 


\subsection{Proposed APT Workflow}

The proposed APT workflow consists of setup, acquisition, and postprocessing stages which can be further augmented with additional sensors and processes to expand the scope of the survey being performed (see system diagram in Figure 2).

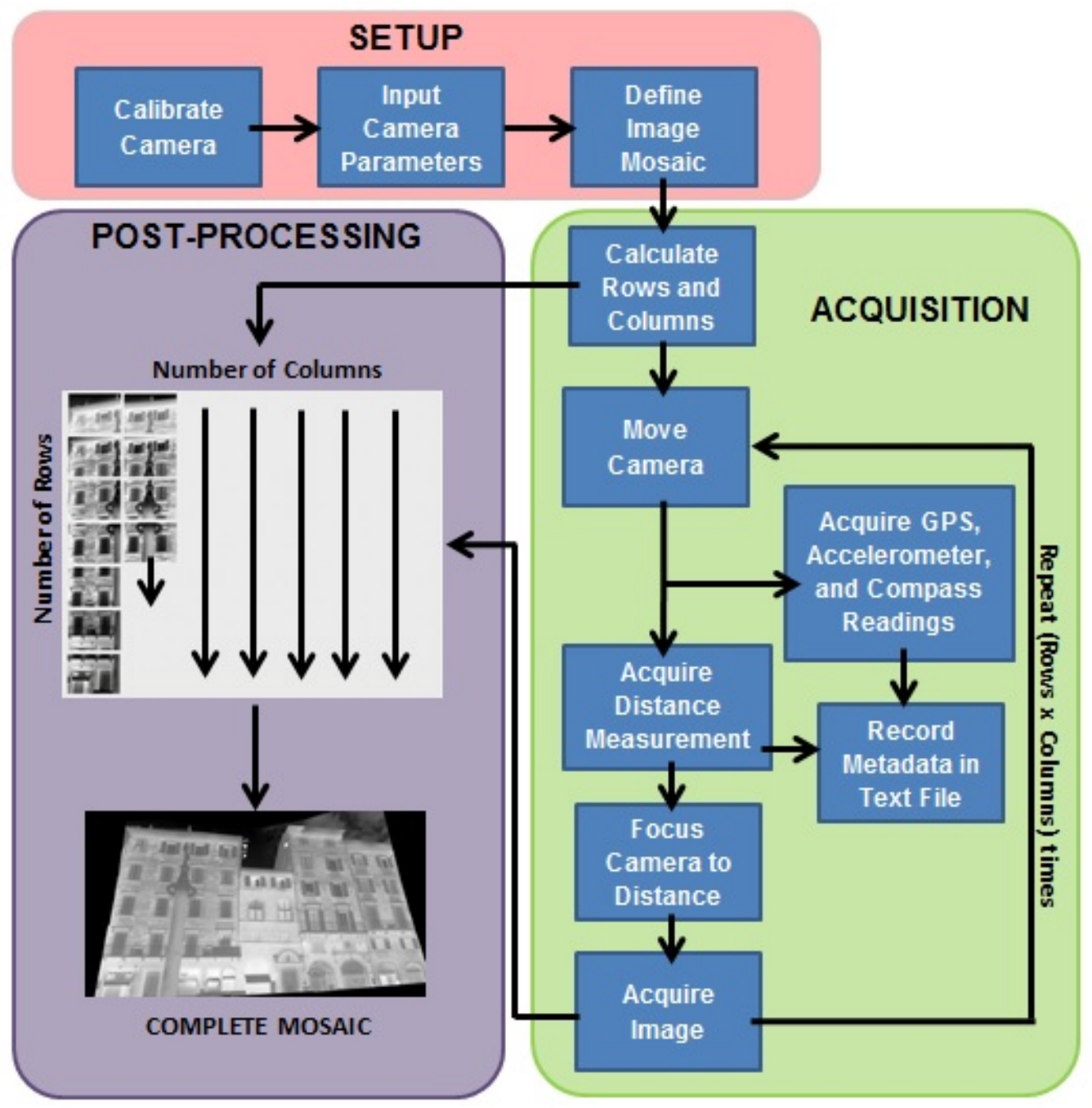

Figure 2: APT Workflow diagram 


\subsubsection{Setup}

In order to obtain accurate and meaningful results, multiple calibration steps are required. The most important initial step is camera calibration which establishes the camera's intrinsic parameters. The calibration provides the camera's lens distortion parameters that aid in the accurate final assembly of the image mosaic, as well as the horizontal and vertical fields of view which are utilized for the initial camera orientation and image recording, resulting in the desired image overlaps.

In addition to the camera calibration and setup, there is the physical setup onsite. The ASTM C1153-10 standard [16] titled Standard Practice for Location of Wet Insulation in Roofing Systems Using Infrared Imaging, discussed by VanOcker et al. [17], provides a reference for recommended operating conditions for passive thermography. Operational specifications such as environmental considerations are helpful in determining when the conditions are best for passive thermal imaging. The operator must establish what is being imaged in order to physically place the imager in a location that will provide a view of the entire target with the desired resolution. Once the camera parameters are known and the imager is in place, the acquisition procedure can be performed.

\subsubsection{Acquisition}

The first step in the acquisition process is to define the region of interest, i.e. the area to be imaged. Once the bounding area is set, the controls system calculates the number of grid positions based on both the desired overlap of the images and the camera parameters. The control system then communicates with the motion platform and camera simultaneously in order to 
acquire images while the platform is stationary at each grid position. While the system is acquiring images it also records metadata and readings from the onboard sensors for subsequent use in image processing and consistent digital reporting. This information can include pan and tilt angles at every image, the distance to the target surface, etc. and can be further augmented with other sensor data. All of this information can prove to be extremely useful in the post-processing of the images and subsequent analysis.

\subsubsection{Post-Processing}

After the acquisition of the images is complete, the camera parameters, relevant metadata and the raw thermal images are used to create the highresolution thermal image mosaic. The first step in post-processing is to correct the raw images for lens distortion as determined in the setup stage. The spatial relationships recorded in the metadata can then guide the placement of the undistorted images. Specifying which images overlap and by how much saves computation time by eliminating the need to check every other image for feature correspondences and only searching for feature correspondences in overlapping areas of the images. Once features detected and matched in all overlapping images, the new composite image can be generated by placing all of the thermal images in their calculated positions and blending them into one high-resolution thermal image.

\subsection{Implemented APT Workflow}

The workflow that was developed to meet the proposed criteria is presented below and is structured such that it can adapt and evolve under specific application scenarios 


\subsubsection{Setup}

The first step in the setup was to establish the thermal camera's intrinsic parameters. Thermal images of a reference checkerboard were acquired from different points of view while using a strong lamp to provide reflections that help to focus the camera and provide contrast in the thermal images. The camera's radial and tangential distortion models were calculated using these checkerboard calibration images [18] and the resulting distortion model, shown in Figure 3, can then be used in the post-processing to undistort all of the thermal images to yield an accurate assembly of images.

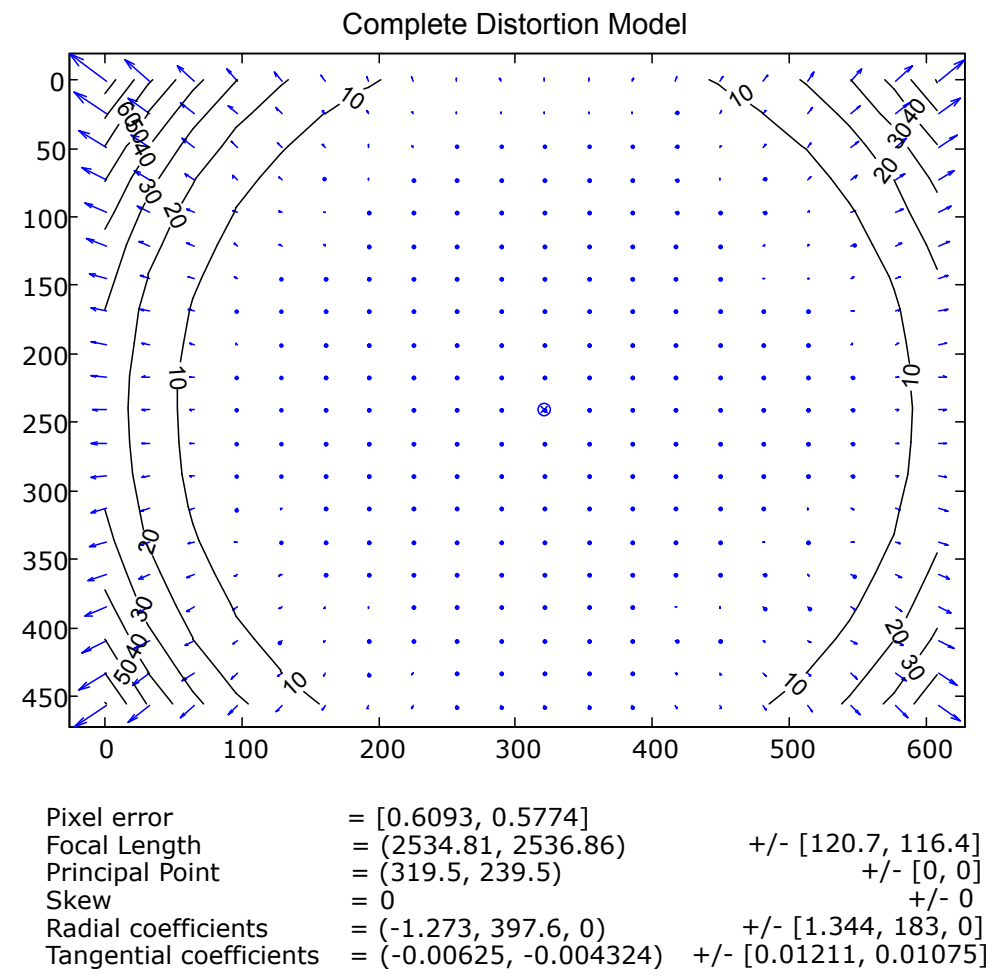

Figure 3: Complete distortion model calculated in MATLAB [18] 
Following the camera calibration came the physical setup on-site. In accordance with the ASTM C1153-10 guidelines [16], imaging was performed in appropriate atmospheric conditions to take advantage of passive environmental thermal gradients. The thermal gradients supply contrast in the thermal images which allows for the detection of anomalies that are present. The imaging equipment was then transported around various sites and positioned in order to acquire complete mosaics of the target structures.

\subsubsection{Acquisition}

In the presented system, the area to be imaged is defined by the user aiming the camera at the top left and bottom right corners of the acquisition area respectively using a graphical user interface. Once the bounding area is set, the number of grid positions is calculated based on the specified overlap of the images and the camera parameters. In the implemented APT system, the acquisition starts at the top left corner and continues down and to the right acquiring images column by column. Based on experimentation in the lab and in the field, overlapping images 33\% in the horizontal direction and $45 \%$ in the vertical was found to be effective. While the system is acquiring images it records metadata and readings from the on-board sensors for subsequent use in image processing and consistent reporting.

The overall speed of the implemented APT system is governed by two steps in the acquisition procedure. The first is the movement of the motion platform which is a function of the types of motors used and the payload weight. The prototype APT platform uses a very economical pan-tilt camera mount (\$350) with servo motors which can be upgraded if faster and more precise results were necessary and if the budget were to allow it. The 
second time intensive aspect of the process is the focusing of the camera. Focusing the camera is a two-step process: distance measurement to the target, followed by operating the camera's motor to refocus to that distance. The acquisition at each image location takes approximately ten seconds in the implemented design, resulting in a panoramic mosaic in a matter of minutes. As the proposed design is small and light, it can easily be moved around a site to thermal site surveys in a short amount of time.

\subsubsection{Post-Processing}

Following acquisition the first post-processing step is to use the distortion model obtained in the setup phase to correct the raw images for lens distortion. In the presented implementation, the raw temperature data is stored in 16-bit thermal images as pixel intensities measured in degrees Kelvin to one one-hundredth of a degree. A problem with the 16-bit images is the lack of feature articulation due to the narrow range of data contained in the images. Initial iterations of thermal image stitching failed due to a lack of features in the raw images.

Computing a successful mosaic first requires contrast enhancement, which rescales the pixel values over the whole range thus losing the raw data contained in the images. This remapping of the image data as well as the grid spacing recorded in the metadata during acquisition allows for automated detection and matching of the newly enhanced features which enables calculation of image positions and orientations [19]. Once the enhanced images are aligned and stitched, the location and orientation of the individual images are recorded as a template and applied to the raw images to recover the raw temperature measurements in the final high-resolution mosaic. 


\section{Case Study: Florence, Italy}

Multiple building-scale thermal surveys of historic landmarks in Florence, Italy were conducted measuring relative temperature gradients in order to study construction details and detect anomalies. As discussed earlier, it is only practical to perform passive thermography at the scale of building surveys, so only environmental heating and cooling is measured. The imaging was performed in the month of October with an average daily high of $21^{\circ} \mathrm{C}$ and average nightly low of $12^{\circ} \mathrm{C}$. The buildings being surveyed are inhabited and therefore internally heated providing an interior heat source to contrast the cold night air. Imaging campaigns were conducted in early mornings when the air temperature was lowest to ensure sufficient thermal gradients for the image mosaics.

The thermal mosaic shown in Figure $4 \mathrm{~b}$ was acquired in the heart of Florence near the famous Santa Maria del Fiore Cathedral focusing on the residential apartments that surround the historical site. The image mosaic is composed of forty-nine images organized into seven rows and seven columns, yielding a five megapixel image. The average spatial resolution is approximately one pixel per square centimeter of the physical building surface which spans thirty-seven meters wide and six stories high. The acquisition of the forty-nine images took ten minutes, averaging twelve seconds per image. In order to capture the same building facade in one image using the same camera and a wide angle lens, the maximum spatial resolution would be thirty square centimeters per pixel, or thirty times less resolution than the presented image mosaic. 


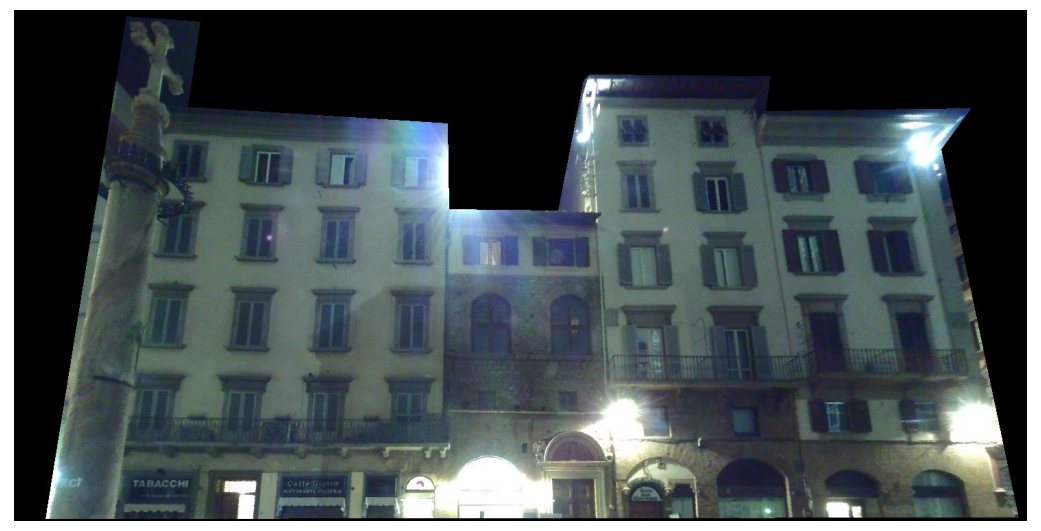

Fig. 4a. Visible image showing the entire building facade

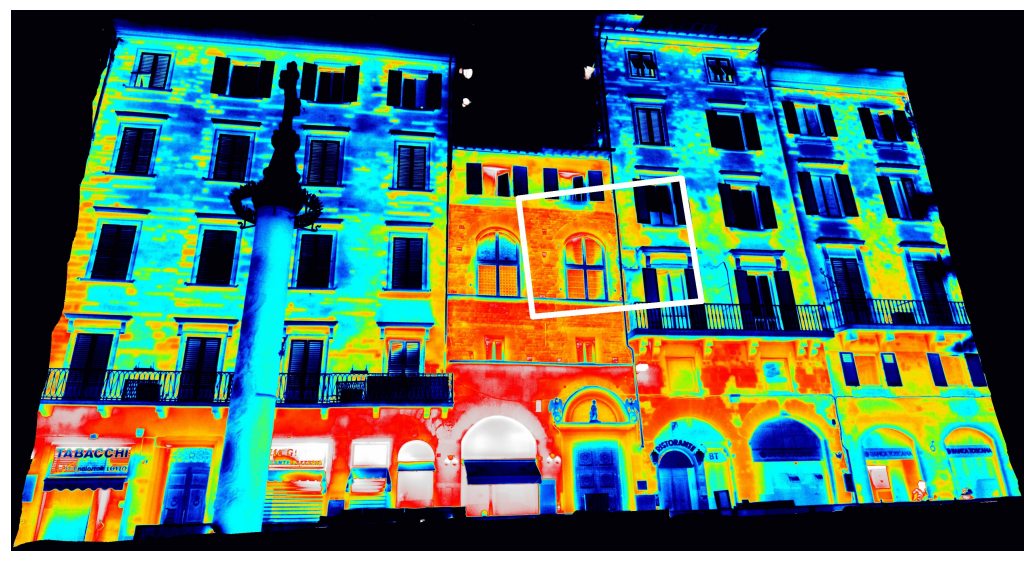

Fig. 4b. Thermal image mosaic showing the entire building facade

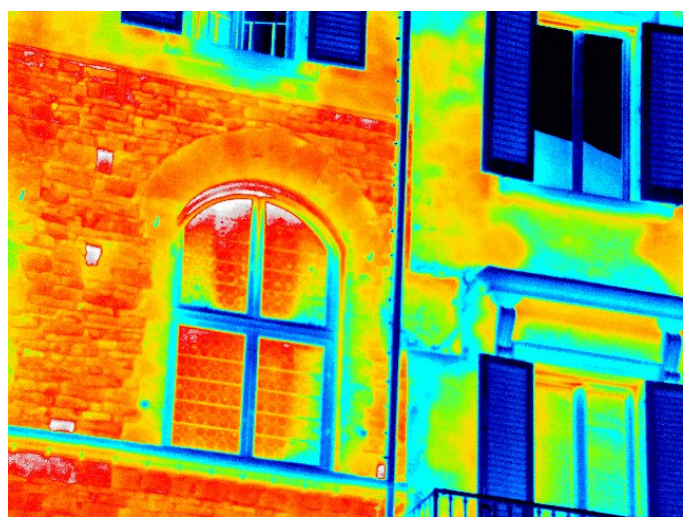

Fig. 4c. Single thermal image

Figure 4: Thermal image mosaic of apartment buildings in Florence, Italy 
Figure $4 \mathrm{a}$ is a visible night-time image of the buildings being surveyed and when compared alongside Figure 4b, differences in construction materials can be observed in the thermal image where they are not normally detectable due to the layer of plaster covering the building's surface. Different types of stone will return a different heat signature and it can be observed, especially below the windows, that stones have been replaced or added at some point in the buildings' histories. Using the full resolution thermal image, individual stones under the plaster layer can be identified. Figure 4c shows an individual thermal image from the mosaic which is outlined in white in Figure $4 \mathrm{~b}$ demonstrating how this automated approach can produce holistic surveys of large buildings while maintaining the fine detail of an individual thermal image.

\section{Conclusion}

This work outlines a new approach for automated high-resolution thermography for the non-destructive evaluation (NDE) of structures. Infrared thermography is an established technology for NDE, but an efficient workflow has yet to be established for the acquisition of thermal image mosaics for building-scale documentation. The presented approach incorporates a cost effective robotic platform, efficient workflow and overall methodology that together enable generation of seamless high-resolution thermal image mosaics which facilitate the detection of small features over an entire building facade. The presented methodology is formed to be repeatable regardless of the user or equipment used, and the workflow also ensures consistent post-processing of the images using the digitally recorded metadata. 
The proposed methodology was successfully deployed in Florence, Italy, generating a multitude of case examples demonstrating its utility. The advantages of this approach are its time efficiency during field acquisitions, post-processing and diagnostic review of the resulting data. When analyzing the thermographic data, it is advantageous to have a holistic, contextual view of the target while maintaining the level of detail required for inspection of small anomalies, instead of scanning through multiple spot surveys. Once the anomaly is observed, it is also much easier to locate and document it in the context of the whole structure instead of a single image with possibly no perspective of location.

The development of an efficient methodology for thermal surveying of large structures is essential in order to monitor the aging built infrastructure. For historic monuments especially, multiple domain experts (structural engineers, architects, historians, restorers, etc.) will often collaborate, contributing the expertise needed to read the data. The presented work demonstrates how trusted data can be acquired to inform the decisions of these trained professionals. This work also demonstrates how automated systems can reduce the time complexity and cost of conducting high-resolution thermal surveys while also establishing consistent digital data recording regardless of the site or operator. The proposed methodology is a comprehensive strategy to increase the capabilities of existing thermal cameras for use in building scale diagnostics. With this methodology in place, it is now possible to pursue a wide range of derivative work, exploring material characterization, damage pattern detection, investigation of construction history, etc. based on the high-resolution data that can now be acquired for large surface areas. 


\section{Acknowledgements}

This work was supported by the National Science Foundation under award \#DGE-0966375, "Training, Research and Education in Engineering for Cultural Heritage Diagnostics," and award \#CNS-1338192, "MRI: Development of Advanced Visualization Instrumentation for the Collaborative Exploration

of Big Data." Additional support was provided by the Qualcomm Institute at UC San Diego, the Friends of CISA3, and the World Cultural Heritage Society. Dimetix FLS-C10 laser distance sensor provided courtesy of DIMETIX USA (www.dimetix-usa.com) - laser distance sensors for industry. Opinions, findings, and conclusions from this study are those of the authors and do not necessarily reflect the opinions of the research sponsors.

\section{References}

[1] C. Balaras, A. Argiriou, Infrared thermography for building diagnostics, Energy and buildings 34 (2) (2002) 171-183.

[2] D. Titman, Applications of thermography in non-destructive testing of structures, NDT \& E International 34 (2) (2001) 149-154.

[3] E. Grinzato, P. Bison, S. Marinetti, Monitoring of ancient buildings by the thermal method, Journal of Cultural Heritage 3 (1) (2002) 21-29.

[4] FLIR Systems Inc., FLIR SC8000 HD series (July 2014).

URL http://www.flir.com/thermography/

[5] GigaPan Systems, Share, play and zoom in on the world through high- 
resolution images (March 2015).

URL http://www.gigapan.com

[6] M. Vollmer, K.-P. Möllmann, Infrared thermal imaging: fundamentals, research and applications, John Wiley \& Sons, 2010.

[7] N. Avdelidis, A. Moropoulou, Applications of infrared thermography for the investigation of historic structures, Journal of Cultural Heritage 5 (1) (2004) 119-127.

[8] P. J. Shull, Nondestructive evaluation: theory, techniques, and applications, CRC press, 2002.

[9] M. Hess, D. Meyer, A. Hoff, D. Rissolo, L. L. Guillermo, F. Kuester, Informing historical preservation with the use of non-destructive diagnostic techniques: A case study at Ecab, Quintana Roo, Mexico, in: Digital Heritage. Progress in Cultural Heritage: Documentation, Preservation, and Protection, Springer, 2014, pp. 659-668.

[10] E. Grinzato, C. Bressan, S. Marinetti, P. Bison, C. Bonacina, Monitoring of the scrovegni chapel by ir thermography: Giotto at infrared, Infrared Physics \& Technology 43 (3) (2002) 165-169.

[11] S. A. Ljungberg, Infrared techniques in buildings and structures: operation and maintenance, Infrared methodology and technology (1994) $211-252$.

[12] M. Hess, F. Kuester, M. Trivedi, Multimodal registration of highresolution thermal image mosaics for the non-destructive evaluation of 
structures, in: Imaging Systems and Techniques (IST), 2014 IEEE International Conference on, IEEE, 2014, pp. 216-221.

[13] S. Imposa, Infrared thermography and georadar techniques applied to the Sala delle Nicchie(Niches Hall) of Palazzo Pitti, Florence (Italy), Journal of Cultural Heritage 11 (3) (2010) 259-264.

[14] Dimetix AG, Dimetix products: FLS laser distance sensor (March 2015). URL http://www.dimetix.com

[15] FLIR Systems Inc., Automation applications infrared camera: FLIR A315 / A615 (July 2014).

URL http://www.flir. com/automation/display/?id=41330

[16] ASTM, ASTM C1153-10 standard practice for location of wet insulation in roofing systems using infrared imaging (2010). doi:10.1520/c1153-10. URL http://dx.doi.org/10.1520/c1153-10

[17] D. A. VanOcker, E. M. Johnson, T. D. Marcotte, The identification of corrosion-related damage from cramp anchors in a limestone-clad building façade using NDE techniques, in: Repair, Retrofit and Inspection of Building Exterior Wall Systems, ASTM International, 2009, pp. 35-3512. doi:10.1520/stp47763s.

URL http://dx.doi.org/10.1520/stp47763s

[18] J.-Y. Bouguet, Camera calibration toolbox for matlab.

[19] New House Internet Services B.V., Photo stitching software 360 degree panorama image software: PTGui.

URL http://www.ptgui.com 